\title{
Influencia de tres ecotipos en la capacidad antioxidante y compuestos fenólicos de harina instantánea de maca (Lepidium meyenii walp)
}

\section{Influence of three echotipes on the antioxidant capacity and phenolic compounds of maca instant flour (Lepidium meyenii walp)}

'Tello Saavedra, R.; Porras Osorio, M.A.L.

\author{
Facultad de Ingeniería en Industrias Alimentarias, Universidad Nacional del Centro del Perú
}

Email: arodotello@yahoo.com

\section{Resumen}

Se determinó la influencia de los ecotipos amarillo, rojo y negro en la capacidad antioxidante y compuestos fenólicos de la harina instantánea de maca. Previamente, se realizó el análisis químico proximal de los ecotipos de maca seca de color negro, amarillo y rojo, encontrándose un nivel de significancia de $\mathrm{P}<0,05$, en los contenidos de humedad, proteína, grasa, fibra y carbohidratos a excepción de las cenizas. Se procedió a elaborar las harinas instantáneas de los tres ecotipos de maca, obteniéndose un rendimiento harinero de $74,53 \%$. Se puso énfasis en las operaciones de extrusión y molienda. Se realizó el análisis químico proximal de las tres harinas instantáneas de maca negra, amarillo y rojo, encontrándose diferencias para un nivel de significancia de $\mathrm{P}<$ 0,05, en humedad, proteína, fibra, cenizas y carbohidratos, excepto las grasas. Las harinas extruidas, presentaron un módulo de finura de 3,0771 en maca amarilla; 3,0215 en maca roja y; 3,0078 en maca negra, predominando las partículas medias. Los análisis físico químicos confirman las características de instantaneidad de las harinas. Los valores de acidez titulable y $\mathrm{pH}$ se encuentran dentro de los valores recomendados para harinas.

Los resultados muestran que los ecotipos de maca, influyen en el contenido de fenoles totales y capacidad antioxidante de las harinas instantáneas de maca, disminuyendo estas en las harinas. Respecto a la capacidad antioxidante, la maca negra, amarilla y roja, presentaron porcentajes de inhibición de radicales libres de $47,83,15,46$ y $28,95 \%$ respectivamente; mientras que en el mismo orden, las harinas instantáneas de maca presentaron 36,25, 14,01 y 27,07 \% de inhibición de radicales libres, notándose que hubo disminución en cada ecotipo. Los fenoles totales para maca negra, amarilla y roja fueron: 289, 364 y $278 \mathrm{mg}$. (GAE/100g). Mientras que en las harinas de maca, de dichos colores, fueron: 239,33, 302,33 y 232,33 respectivamente. El análisis estadístico muestra que existe diferencia significativa entre las medias de los tratamientos a un nivel de $95 \%$ de probabilidad.

Palabras Clave: harina instantánea de maca, ecotipos, fenoles totales, capacidad antioxidante

\section{Abstract}

The influence of the yellow, red and black ecotypes on the antioxidant capacity and phenolic compounds of maca instant flour was determined. Previously, the chemical analysis of the ecotypes of dry black, yellow and red maca was carried out, finding significant differences for a level of significance of $\mathrm{P}<0.05$, in the contents of moisture, protein, fat, fiber and carbohydrates, but the ashes. Instant flours yields $74.53 \%$ of the three ecotypes of maca. Emphasis was placed on extrusion and milling operations. The proximal chemical analysis of instant flours of black, yellow and red maca was carried out, finding significant differences for a level of significance of $\mathrm{P}<0.05$, in moisture, protein, fiber, ashes and carbohydrates, except fats. The extruded flours presented a fineness modulus of 3.0771 in yellow maca; 3.0215 in red maca and 3.0078 in black maca, prevailing the average particles. The physical-chemical analyzes confirm the instantaniety characteristics of the flours. The titratable acidity values and $\mathrm{pH}$ are within the recommended values for flours.

The results show that the maca ecotypes influence the content of total phenols and antioxidant capacity of the instantaneous maca flours, decreasing these in flours. Regarding the antioxidant capacity, the black, yellow and red maca presented percentages of free radical inhibition of $47.83,15.46$ and $28.95 \%$ respectively, while the instant flours of black, yellow and red maca presented 36, 25, 14.01 and $27.07 \%$ inhibition of free radicals respectively, noting that there was a decrease in each ecotype. The total phenols for black, yellow and red maca were: 289, 364 and $278 \mathrm{mg}$. GAE / 100g), while in black, yellow and red maca flours were: 239.33, 302.33 and 232.33 respectively. The statistical analysis shows that there is a significant difference between the means of the treatments at a 95\% probability level.

Keywords: instant maca meal, ecotypes, total phenols, antioxidant capacity 


\section{Introducción}

La maca presenta metabolitos secundarios como glicosinolatos (Yábar et al., 2011) y compuestos fenólicos (Campos et al., 2013) lo que ha conllevado a realizar diversos estudios donde se evidencian las propiedades antioxidantes (Gonzáles et al., 2006) y otras propiedades biológicas en el área médica, por lo que su consumo genera un impacto positivo en la salud de las personas; razón por la cual, el interés comercial de esta especie ha ido aumentando progresivamente (Sun et al., 2008).

Utilizando el ensayo de DPPH, se analizaron las propiedades antioxidantes en aceites esenciales, lípidos y polisacáridos de maca amarilla, roja y negra. La correlación de las composiciones principales y las actividades antioxidantes se evaluó mediante un método estadístico (Sun et al., 2008).

Se estudió el efecto de los tratamientos a corto y largo plazo con tres ecotipos de Lepidium meyenii en espermatogénesis en ratas, concluyéndose que existe diferencias en la respuesta biológica de los ecotipos amarillo, rojo y negro, destacándose que la maca negra puede tener mayor efecto benéfico en el recuento de espermatozoides y la motilidad del mismo (Sun et al., 2008).

La maca es conocida como una planta peruana alto valor nutricional, funcional y medicinal, debido a las propiedades que presenta tales como, mejorar el aprendizaje la función sexual y la espermatogénesis, así como la reducción del estrés, entre otras propiedades, las mismas que podrían estar relacionadas con la variedad de este hipocótilo. Sin embargo, estas propiedades no sólo están basadas en el ecotipo o el color sino también en el tipo de prácticas del procesamiento de la maca (Troya-Santos, 2017).

Se ha observado que en el mercado las harinas más comerciales son la de maca cruda e instantánea de maca, las cuales se exportan fundamentalmente en cantidades significativas a los mercados de Japón y Estados Unidos (Durand et al., 2004). Por lo tanto, se presume que existe variación en el contenido de compuestos fenólicos y capacidad antioxidante de la harina instantánea influenciadas por los ecotipos de maca. Si bien la composición química del hipocotilo puede ser afectada por su color, pocos estudios han comparado la capacidad antioxidante y compuestos fenólicos de los ecotipos amarillo, rojo y negro en harinas instantáneas para su consumo diferenciado.

La maca es considerada un producto bandera del Perú y, por tanto, es de interés de la comunidad científica, los consumidores y los potenciales consumidores, saber cuál de los ecotipos amarillo, rojo y negro tiene mayor capacidad antioxidante y contenido de compuestos fenólicos y cómo influyen en la harina instantánea de maca.

Si la composición química del hipocotilo puede ser afec- tada por su color, entonces este es un factor que va a influenciar en la capacidad antioxidante y compuestos fenólicos de la harina instantánea de maca elaborada con los ecotipos amarillo, rojo y negro.

Si ya se tienen estudios nutricionales y clínicos del efecto de la maca sobre las diversas funciones en el organismo humano, tanto en extractos, aceites esenciales y en los diversos estadíos de la planta, es necesario también conocer la influencia que tienen los ecotipos de maca en las propiedades antioxidantes de la harina instantánea para que los consumidores puedan elegir el producto que pueda retardar mejor el envejecimiento.

El objetivo de la investigación fue determinar la influencia de los ecotipos amarillo, rojo y negro en la capacidad antioxidante y compuestos fenólicos de la harina instantánea de maca. Si bien es cierto que los antioxidantes naturales presentes en alimentos como la maca nos protegen de los radicales libres, que son los que causan enfermedades y envejecimiento, es también cierto que está contraindicado para personas que sufren de la presión alta y es necesario estudiar efectos a corto y mediano plazo en humanos. Los objetivos específicos fueron: Determinar la composición químico proximal, capacidad antioxidante y compuestos fenólicos de los ecotipos de maca amarilla, rojo y negro. Realizar la caracterización físico química de las harinas instantáneas de ecotipos de maca amarillo, rojo y negro y determinar la capacidad antioxidante y compuestos fenólicos de harinas instantáneas de ecotipos de maca amarillo, rojo y negro.

\section{Materiales y métodos}

Ecotipos de maca amarillo, rojo y negro fueron analizados en los laboratorios de análisis instrumental y control de calidad de la Facultad de Ingeniería en Industrias Alimentarias de la Universidad Nacional del Centro del Perú, ubicado en el distrito de El Tambo, provincia de Huancayo. Para esta investigación, se tomaron muestras al azar de maca seca de los ecotipos amarillo rojo y negro, las mismas que fueron recolectadas de parcelas ubicadas en la localidad de Junín provincia de Junín, posteriormente fueron analizadas y procesadas para obtener la harina instantánea de maca de cada ecotipo. Se realizó el análisis físico químico, determinación de la capacidad antioxidante y compuestos fenólicos de los ecotipos y harinas.

Esta investigación se inició en el mes de marzo del 2018 y se culminó en el mes de diciembre del mismo año. Se trabajó con una muestra de $30 \mathrm{~kg}$ de los ecotipos de maca seca distribuyéndolos de la siguiente manera: 10 $\mathrm{kg}$ de ecotipo amarillo, $10 \mathrm{~kg}$ de ecotipo rojo y $10 \mathrm{~kg}$ de ecotipo negro, los cuales fueron evaluados mediante análisis físicos químicos, determinación de actividad antioxidante y compuestos fenólicos. Luego se procedió a elaborar las harinas instantáneas de maca de los 3 eco- 
tipos mediante el diagrama de flujo que se presenta en la Figura 1. Se realizaron análisis físicos químicos, determinación de actividad antioxidante y compuestos fenólicos de las harinas de cada ecotipo.

\section{Figura 1}

Diagrama de flujo del proceso de elaboración de harina de maca (Lepidium meyenii walp) instantánea.

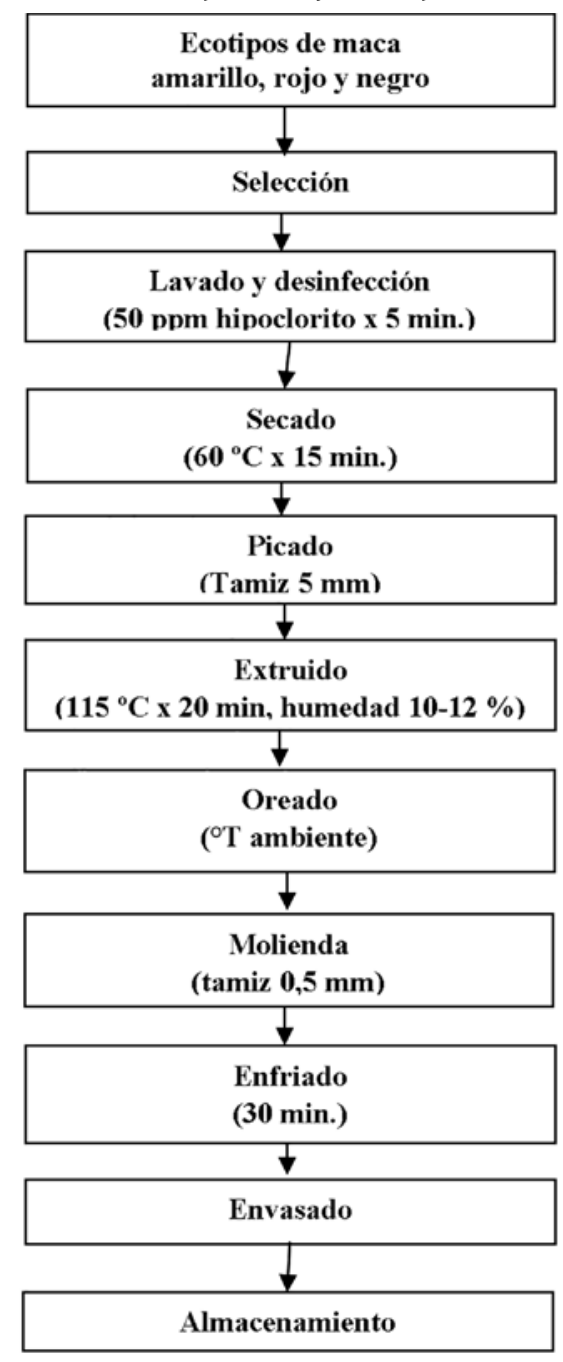

El número de muestras analizadas fueron seis $(n=6)$, extraídas de una población de maca orgánica seca de los ecotipos amarillo, rojo y negro, proporcionado por la Asociación de Productores de Maca del Valle del Mantaro, recolectados de parcelas ubicadas en la localidad de Junín, provincia de Junín. Las muestras fueron tomadas al azar a partir del lote cosechado de las parcelas antes referidas. Se tomó una muestra de $30 \mathrm{~kg}$. de maca seca. De esta muestra se tomó una sub muestra de $10 \mathrm{~kg}$. por cada ecotipo (amarillo, rojo y negro) para la elaboración de las harinas instantáneas. Los treinta kilogramos de maca de los 3 ecotipos estudiados, fueron seleccionados de todo el conjunto de la cosecha, separando los que no corresponden a los ecotipos en estudio.

Los ecotipos de maca estudiados fueron evaluados mediante los análisis de laboratorio que se detallan a continuación:

\section{Análisis químico proximal}

Humedad.- Según métodos oficiales de análisis de la Association of oficial Analytical Chemists (AOAC, 1995)

Proteínas.- Se determina por el método de micro kjeldahl, propuesto por (AOAC, 1995).

Grasa.- Método de extracción con solvente (Soxhlet), propuesto por (AOAC, 1995).

Fibra. - Mediante una hidrólisis ácida y alcalina, método recomendado por (AOAC, 1995).

Cenizas.- Método de calcinación directa de la muestra en horno mufla a $600^{\circ} \mathrm{C}$, según método (AOAC, 1995).

Carbohidratos.- Se determinó por diferencia de 100 y la suma de los otros componentes (humedad, proteínas, grasa, fibra, cenizas y carbohidratos), método (AOAC, 1995).

\section{Análisis físico químico}

\section{Determinación de acidez titulable y pH}

La acidez titulable se halla siguiendo las pautas del método aplicable a harinas del INDECOPI (INDECOPI, 1998), que consiste en pesar 10 grs. de harina y se lleva a un erlenmeyer de $300 \mathrm{ml}$ y se diluye con $100 \mathrm{ml}$ de agua destilada. Luego se agitó la suspensión contenida en el frasco cada 10 minutos por espacio de una hora. Se filtró la suspensión utilizando un filtro de porosidad intermedia, hasta obtener un volumen superior a los $50 \mathrm{ml}$.

Se tomó $50 \mathrm{ml}$ del filtrado y se lleva a un erlenmeyer de $125 \mathrm{ml}$ añadiendo $1 \mathrm{ml}$ de solución de fenolftaleína (indicador) al $1 \%$. Se titula con una solución de $\mathrm{NaOH} 0.1$ $\mathrm{N}$ hasta que produzca un viraje a color grosella, debiendo persistir por espacio de 30 segundos, seguidamente se anota el gasto de soda. El resultado se expresa en \% referido al ácido sulfúrico.

La determinación de $\mathrm{pH}$ se efectúa en un pH metro digital ( $\mathrm{pH} 0$ - 14), para lo cual se usa una solución filtrada obtenida siguiendo el procedimiento referido para la acidez titulable.

\section{Análisis granulométrico}

Este análisis se efectuó con la finalidad de determinar la eficiencia de la molienda en las harinas instantáneas de maca a través de la determinación del módulo de finura de las partículas. El procedimiento para efectuar el análisis granulométrico, consiste en colocar y ordenar los tamices de espesores de mallas diferentes en orden ascendente de tal manera que el tamiz de abertura de malla más gruesa quede en la parte superior, luego pesar $100 \mathrm{~g}$ de harina y colocarlo en el tamiz superior (el más grueso); luego someter a agitación o zarandear durante 10 a 15 minutos todo el sistema de tamices para que cada tamiz deje pasar las partículas de acuerdo a su abertura y; por último, pesar las partículas retenidas en cada ta- 
miz y llevarlo a porcentaje de material retenido en cada tamiz para realizar los cálculos correspondientes. La clasificación, según el INDECOPI, de acuerdo al módulo de finura de las harinas es la siguiente: 0 - 2 = Fino; 2 - 4 = Medio; 4 a más = Grueso (Pascual y Ramos, 2000).

\section{Índice de absorción de agua}

El método seguido para esta determinación, consiste en pesar 2.5 gramos de muestra de harina a la que se le agrega $30 \mathrm{ml}$. de agua a $30{ }^{\circ} \mathrm{C}$ (Se pasa en los tubos de centrifuga previamente tarados). Se somete a agitación intermitente por $30 \mathrm{~min}$., luego de los cuales se coloca en una centrífuga de $3000 \mathrm{rpm}$. por $10 \mathrm{~min}$. El supernadante se pasa a un vasito previamente tarado. Se toma el peso del gel (Vivas, 1979).

$$
\text { Índice de absorción }=\frac{\text { gramos de gel }}{\text { gramos de muestra }}
$$

\section{Índice de solubilidad}

El método seguido para esta determinación fue el método descrito por Vivas (1979). El supernadante que ha recibido el vaso de precipitado, se lleva a calentar en una estufa a $90{ }^{\circ} \mathrm{C}$., a fin de evaporar y concentrar el supernadante. Se determina el peso de sólidos solubles y se expresa en \% de los 2.5 gr. de muestra.

\section{Determinación de polifenoles totales}

Para la determinación del contenido de fenoles totales presente en los distintos extractos de maca se empleó el método espectrofotométrico reportado por Singleton y Rossi (1965), empleando folin - ciocalteau como agente reductor para la determinación de fenoles totales. La absorbancia del color azul desarrollado se mide a $765 \mathrm{~nm}$ en un espectrofotómetro UV-VIS Shimadzu. El estándar utilizado fue el ácido gálico. Los resultados se expresaron en mg ácido gálico equivalente/ $100 \mathrm{~g}$ muestra.

\section{Determinación de la capacidad antioxidante}

Se empleó el ensayo DPPH (Brand-Williams et al., 1995) basado en la reacción con el 2,2 difenil-1-picrilhidrazilo (DPPH). Este ensayo está fundamentado en la reducción del radical DPPH por los antioxidantes que contiene la muestra. Se utiliza una longitud de onda de $515 \mathrm{~nm}$ para registrar la lectura de la absorbancia utilizando un espectrofotómetro Shimadzu. Los resultados de la actividad antioxidante se expresaron en porcentaje de inhibición de radicales libres.

\section{Análisis estadístico}

Se calcularon los promedios y desviación estándar de los componentes del análisis químico proximal (humedad, proteína, grasa, fibra, ceniza y carbohidratos) de los ecotipos y harinas instantáneas. Los cálculos reportan el promedio de 3 repeticiones por ecotipo y harina y la desviación estándar de la media. Promedio y desviación estándar de las determinaciones de capacidad antioxidante (\% de inhibición de radicales libres) y polifenoles totales (mg GAE/100 g) de los ecotipos y harinas instantáneas.

Análisis de varianza y prueba de Tukey de la capacidad antioxidante de ecotipos y harinas instantáneas de maca

Los componentes del análisis químico de los ecotipos y harinas, fueron analizados estadísticamente a través de un análisis de variancia (ANOVA) y de existir diferencias estadísticas entre los tratamientos se aplicó la prueba de significación de Tukey, para un nivel de significancia de $\mathrm{P}<0,05$. Se utilizó un diseño completamente al azar (DCA). Este mismo diseño estadístico (DCA), se utilizó para el análisis de la capacidad antioxidante y compuestos fenólicos de los ecotipos y harinas de maca obtenidos en la investigación. Cada componente se analizó con 3 repeticiones por análisis. Se utilizó el programa IBM SPSS Statistic versión 21, para el procesamiento y análisis de datos.

\section{Resultados}

\section{Controles fisicoquímicos}

\section{Análisis químico-proximal de los tres ecotipos de maca}

En la Tabla 1, se presenta los resultados promedios y la desviación estándar de la media del análisis químico proximal de 3 ecotipos de maca seca, utilizados en la investigación. Se observa algunas variaciones en el contenido de humedad, proteínas, grasa, fibra y carbohidratos a excepción de cenizas.

Tabla 1

Resultados promedios del análisis químico proximal de 3 ecotipos de maca seca ( $\mathrm{g} / 100 \mathrm{~g}$ de muestra)

\begin{tabular}{lccc}
\hline \multirow{2}{*}{ Componentes } & \multicolumn{3}{c}{ Ecotipos de maca } \\
\cline { 2 - 4 } & $\begin{array}{c}\text { Maca Ecotipo Maca Ecotipo } \\
\text { Negro (T1) }\end{array}$ & $\begin{array}{c}\text { Maca Ecotipo } \\
\text { Rojillo (T2) }\end{array}$ & Rojo (T3) \\
\cline { 2 - 4 } & $\overline{\mathbf{x}} \pm \mathbf{D E})$ & $(\overline{\mathbf{x}} \pm \mathbf{D E})$ & $(\overline{\mathbf{x}} \pm \mathbf{D E})$ \\
\hline Humedad & $9,10 \pm 0,14^{\mathrm{c}}$ & $9,87 \pm 0,03^{\mathrm{b}}$ & $14,75 \pm 0,24^{\mathrm{a}}$ \\
Proteína & $12,78 \pm 0,04^{\mathrm{a}}$ & $11,76 \pm 0,03^{\mathrm{b}}$ & $12,74 \pm 0,07^{\mathrm{a}}$ \\
Grasa & $0,28 \pm 0,01^{\mathrm{b}}$ & $0,38 \pm 0,02^{\mathrm{a}}$ & $0,29 \pm 0,01^{\mathrm{b}}$ \\
Fibra & $4,88 \pm 0,11^{\mathrm{a}}$ & $4,88 \pm 0,15^{\mathrm{a}}$ & $3,97 \pm 0,04^{\mathrm{b}}$ \\
Ceniza & $5,47 \pm 0,14^{\mathrm{a}}$ & $5,32 \pm 0,03^{\mathrm{a}}$ & $5,42 \pm 0,04^{\mathrm{a}}$ \\
Carbohidratos & $67,49 \pm 0,11^{\mathrm{b}}$ & $67,99 \pm 0,17^{\mathrm{a}}$ & $62,83 \pm 0,04^{\mathrm{c}}$ \\
\hline
\end{tabular}

Nota. Los resultados son el promedio de 3 repeticiones por ecotipo y la desviación estándar de la media. Se realizó el ANOVA de un factor y la prueba de diferencia de medias de Tukey (post hoc) y, por tanto, las letras distintas en una misma fila denotan diferencia significativa $(p<0,05)$. 
Análisis químico-proximal de las harinas instantáneas de tres ecotipos de maca

En la Tabla 2, se presenta los resultados promedios y la desviación estándar de la media del análisis químico proximal de las harinas instantáneas de 3 ecotipos de maca. Nótese algunas variaciones en el contenido de humedad, proteínas, fibra cruda, cenizas y carbohidratos a excepción de grasas.

Tabla 2

Resultados promedios del análisis químico proximal de harinas instantáneas de ecotipos de maca ( $\mathrm{g} / 100 \mathrm{~g}$ de muestra)

\begin{tabular}{lccc}
\hline \multicolumn{4}{c}{ Harinas instantáneas de ecotipos de maca } \\
\hline Componentes & $\begin{array}{c}\text { Harina } \\
\text { instantánea } \\
\text { de maca } \\
\text { negra (T4) }\end{array}$ & $\begin{array}{c}\text { Harina } \\
\text { instantánea } \\
\text { de maca } \\
\text { amarilla (T5) }\end{array}$ & $\begin{array}{c}\text { Harina } \\
\text { instantánea } \\
\text { de maca } \\
\text { rojo (T6) }\end{array}$ \\
\cline { 2 - 4 } & $\mathbf{( \overline { \mathbf { x } } \pm \mathbf { D E } )}$ & $\mathbf{( \overline { \mathbf { x } } \pm \mathbf { D E } )}$ & $\mathbf{( \overline { \mathbf { x } } \pm \mathbf { D E } )}$ \\
\hline Humedad & $4,75 \pm 0,05^{\mathrm{c}}$ & $5,02 \pm 0,03^{\mathrm{b}}$ & $5,31 \pm 0,03^{\mathrm{a}}$ \\
Proteínas & $11,59 \pm 0,03^{\mathrm{a}}$ & $10,29 \pm 0,03^{\mathrm{c}}$ & $11,30 \pm 0,03^{\mathrm{b}}$ \\
Grasa & $0,20 \pm 0,02^{\mathrm{a}}$ & $0,31 \pm 0,01^{\mathrm{a}}$ & $0,88 \pm 1,17^{\mathrm{a}}$ \\
Fibra cruda & $4,00 \pm 0,01^{\mathrm{a}}$ & $3,87 \pm 0,11^{\mathrm{a}}$ & $3,02 \pm 0,02^{\mathrm{b}}$ \\
Cenizas & $3,46 \pm 0,05^{\mathrm{a}}$ & $3,11 \pm 0,10^{\mathrm{b}}$ & $3,20 \pm 0,01^{\mathrm{b}}$ \\
Carbohidratos & $75,00 \pm 0,08^{\mathrm{b}}$ & $77,41 \pm 0,10^{\mathrm{a}}$ & $76,28 \pm 1,17^{\mathrm{ab}}$ \\
\hline
\end{tabular}

Nota: Los resultados son el promedio de 3 repeticiones por harina y la desviación estándar de la media. Se realizó el ANOVA de un factor y la prueba de diferencia de medias de Tukey (post hoc) $\mathrm{y}$, por tanto, las letras distintas en una misma fila denotan diferencia significativa $(\mathrm{p}<0,05)$. Se utilizó el programa SPSSV 21.

Análisis físico químico de harinas instantáneas de ecotipos de maca

En la Tabla 3, se presenta los resultados promedios y la desviación estándar de la media del análisis físico químico de las harinas instantáneas de tres ecotipos de maca en 100 g. de muestra. Los índices de solubilidad y absorción muestran sus características de instantaneidad y fácil rehidratación.

Tabla 3

Resultados promedios del análisis físico químico de las harinas extruidas de tres ecotipos de maca (en 100g. de muestra)

\begin{tabular}{lccc}
\hline \multicolumn{1}{c}{ Análisis } & $\begin{array}{c}\text { Harina extru-Harina extru- } \\
\text { ida ecotipo } \\
\text { amarillo }\end{array}$ & $\begin{array}{c}\text { Harina ex- } \\
\text { ida ecotipo } \\
\text { rojo }\end{array}$ & $\begin{array}{c}\text { truída ecotipo } \\
\text { negro }\end{array}$ \\
\cline { 2 - 4 } & $(\overline{\mathbf{x}} \pm \mathbf{D E})$ & $(\overline{\mathbf{x}} \pm \mathbf{D E})$ & $(\overline{\mathbf{x}} \pm \mathbf{D E})$ \\
\hline $\begin{array}{l}\text { Índice de solubil- } \\
\text { idad (\%) }\end{array}$ & $14,4 \pm 0.20$ & $14,2 \pm 0.20$ & $13,9 \pm 0.26$ \\
$\begin{array}{l}\text { Índice de } \\
\text { absorción de agua } \\
\text { (g. gel/g. muestra) }\end{array}$ & $4,3 \pm 0.20$ & $4,2 \pm 0.20$ & $4,2 \pm 0.10$ \\
$\begin{array}{l}\text { Acidez Titulable } \\
(\% \text { ácido sulfúrico) }\end{array}$ & $0,38 \pm 0.02$ & $0,36 \pm 0.02$ & $0,40 \pm 0.02$ \\
pH a $20{ }^{\circ} \mathrm{C}$ & $5,56 \pm 0.02$ & $5,69 \pm 0.01$ & $5,43 \pm 0.02$ \\
\hline
\end{tabular}

Nota: Los resultados son el promedio de 3 repeticiones por harina y la desviación estándar de la media.
Análisis granulométrico de harinas instantáneas de ecotipos de maca

En las tablas 4, 5 y 6, se da a conocer los resultados del análisis granulométrico de las Harinas de Maca extruida de los tres ecotipos estudiados a través de la determinación del módulo de finura y el análisis granulométrico acumulado (AGA). Nótese que el tamaño de partículas presentes en mayor proporción en las harinas instantáneas de maca corresponde a los tamices números 30, 40 y 50 que tienen una abertura de malla de 600, 425 y 300 micras, respectivamente.

Tabla 4

Análisis granulométrico - Determinación del módulo de finura de la harina extruida de maca amarilla

\begin{tabular}{cccccc}
\hline \multirow{2}{*}{$\begin{array}{c}\text { Número } \\
\text { de tamiz }\end{array}$} & $\begin{array}{c}\text { Abertura } \\
\text { de la malla } \\
\text { (micras) }\end{array}$ & $\begin{array}{c}\text { Material } \\
\text { retenido } \\
\text { (\%) }\end{array}$ & Factor & \multicolumn{4}{c}{$\begin{array}{c}\text { Harina } \\
\text { extruida de } \\
\text { maca } \\
\text { amarilla }\end{array}$} & AGA \\
\hline 30 & 600 & 46,35 & 4 & 185,40 & 100,00 \\
40 & 425 & 31,87 & 3 & 95,61 & 53,65 \\
50 & 300 & 10,16 & 2 & 20,32 & 21,78 \\
60 & 250 & 6,38 & 1 & 6,38 & 11,62 \\
Plato & ---- & 5,24 & -- & --- & 5,24 \\
& & 100,00 & & 307,71 & \\
\hline
\end{tabular}

Nota: Módulo de finura $=307,71 / 100=3,0771$

Tabla 5

Análisis granulométrico - Determinación del módulo de finura de la harina extruida de maca roja

\begin{tabular}{cccccc}
\hline $\begin{array}{c}\text { Número } \\
\text { de tamiz }\end{array}$ & $\begin{array}{c}\text { Abertura } \\
\text { de la malla } \\
\text { (micras) }\end{array}$ & $\begin{array}{c}\text { Material } \\
\text { retenido } \\
(\%)\end{array}$ & Factor & $\begin{array}{c}\text { Harina } \\
\text { extruida de } \\
\text { maca roja }\end{array}$ & AGA \\
\hline 30 & 600 & 43,95 & 4 & 175,80 & 100,00 \\
40 & 425 & 32,20 & 3 & 96,60 & 56,05 \\
50 & 300 & 10,96 & 2 & 21,92 & 23,85 \\
60 & 250 & 7,83 & 1 & 7,83 & 12,89 \\
Plato & ---- & 5,06 & -- & ---- & 5,06 \\
\hline Total & & 100,00 & & 302,15 & \\
\hline
\end{tabular}

Nota: Módulo de finura $=302,15 / 100=3,0215$

Tabla 6

Análisis granulométrico - Determinación del módulo de finura de la harina extruida de maca negra

\begin{tabular}{|c|c|c|c|c|c|}
\hline $\begin{array}{l}\text { Número } \\
\text { de tamiz }\end{array}$ & $\begin{array}{c}\text { Abertura } \\
\text { de la malla } \\
\text { (micras) }\end{array}$ & $\begin{array}{c}\text { Material } \\
\text { retenido } \\
(\%)\end{array}$ & Factor & $\begin{array}{c}\text { Harina } \\
\text { extruida } \\
\text { de maca } \\
\text { negra }\end{array}$ & AGA \\
\hline 30 & 600 & 44,18 & 4 & 176,72 & 100,00 \\
\hline 40 & 425 & 31,28 & 3 & 93,84 & 55,82 \\
\hline 50 & 300 & 11,13 & 2 & 22,26 & 24,54 \\
\hline 60 & 250 & 7,96 & 1 & 7,96 & 13,41 \\
\hline Plato & ---- & 5,45 & -- & ---- & 5,45 \\
\hline Total & & 100,00 & & 300,78 & \\
\hline
\end{tabular}

Nota: Módulo de finura $=300,78 / 100=3,0078$ 
Determinación de la capacidad antioxidante de ecotipos y harinas instantáneas de maca

En la Tabla 7, se presenta los resultados promedios obtenidos en la determinación de la capacidad antioxidante de los tres ecotipos y harinas instantáneas de maca, expresadas en porcentaje de inhibición de radicales libres. Así mismo, se presenta de maca, expresadas en porcentaje de inhibición de la desviación estándar de la media, obtenida de 3 repeticiones por ecotipo y harina.

Tabla 7

Determinación de la capacidad antioxidante de los tres ecotipos y harinas instantáneas de maca (\% inhibición de radicales libres)

\begin{tabular}{lcc}
\hline \multicolumn{1}{c}{$\begin{array}{c}\text { Ecotipos y harinas } \\
\text { instantáneas de maca }\end{array}$} & \multicolumn{2}{c}{$\begin{array}{c}\text { Capacidad antioxidante } \\
\text { (\% de inhibición de } \\
\text { radicales libres) }\end{array}$} \\
\cline { 2 - 3 } Maca ecotipo negra & Tratamientos & $\mathbf{( \overline { \mathbf { x } } \pm \mathbf { D E } )}$ \\
Maca ecotipo amarilla & $\mathrm{T} 2$ & $47,83 \pm 0,08$ \\
Maca ecotipo roja & $\mathrm{T} 3$ & $28,95 \pm 0,07$ \\
Harina instantánea de maca negra & $\mathrm{T} 4$ & $36,25 \pm 0,06$ \\
Harina instántánea de maca amarilla & $\mathrm{T} 5$ & $14,01 \pm 0,02$ \\
Harina instantánea de maca rojo & $\mathrm{T} 6$ & $27,07 \pm 0,06$ \\
\hline
\end{tabular}

Nota: Los resultados son el promedio de tres repeticiones por ecotipo y harina y la desviación estándar de la media

En la Figura 2, se presenta la representación gráfica del análisis de variancia y prueba de Tukey de la capacidad antioxidante de ecotipos y harinas instantáneas de maca. Se observa diferencias significativas a nivel de ecotipos y harinas instantáneas de maca.

\section{Figura 2}

Representación gráfica del análisis de variancia y prueba de Tukey de la capacidad antioxidante de ecotipos y harinas instantáneas de maca $(\mathrm{p}<0.05)$.

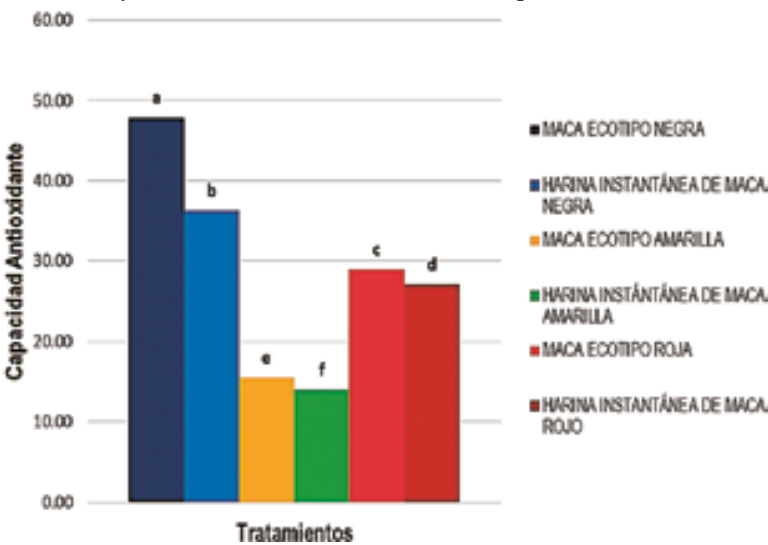

Nota. Letras distintas en una misma barra denotan diferencia significativa al $95 \%$ de nivel de confianza.

En la Tabla 8, se presenta los resultados promedios obtenidos en la determinación de polifenoles totales de los tres ecotipos y harinas instantáneas de maca, expresadas en mg. de ácido gálico equivalente por 100 gramos de muestra (mg GAE/100 g). Así mismo, se presenta la desviación estándar de la media, obtenida de 3 repeticiones por ecotipo y harina.

\section{Tabla 8}

Determinación de la polifenoles totales de los tres ecotipos y harinas instantáneas de maca (mg GAE/100 g)

\begin{tabular}{lcc}
\hline \multicolumn{1}{c}{$\begin{array}{c}\text { Ecotipos y harinas } \\
\text { instantáneas de maca }\end{array}$} & \multicolumn{2}{c}{$\begin{array}{c}\text { Capacidad antioxidante } \\
\text { (\% de inhibición de } \\
\text { radicales libres) }\end{array}$} \\
\cline { 2 - 3 } Maca ecotipo negra & Tratamientos & $(\overline{\mathbf{x}} \pm \mathbf{D E})$ \\
Maca ecotipo amarilla & $\mathrm{T} 2$ & $289 \pm 1,00$ \\
Maca ecotipo roja & $\mathrm{T} 3$ & $278 \pm 4,58$ \\
Harina instantánea de maca negra & $\mathrm{T} 4$ & $239,33 \pm 0,58$ \\
Harina instantánea de maca amarilla & $\mathrm{T} 5$ & $302,33 \pm 2,52$ \\
Harina instantánea de maca rojo & $\mathrm{T} 6$ & $232,33 \pm 2,52$ \\
\hline
\end{tabular}

Nota: Los resultados son el promedio de tres repeticiones por ecotipo y harina y la desviación estándar de la media

En la Figura 3, se presenta la representación gráfica del análisis de variancia y prueba de Tukey de la capacidad antioxidante de ecotipos y harinas instantáneas de maca. Nótese las diferencias significativas a nivel de ecotipos y harinas instantáneas de maca.

\section{Figura 3}

Representación gráfica del análisis de variancia y prueba de Tukey del contenido de fenoles totales de ecotipos y harinas instantáneas de maca $(\mathrm{p}<0.05)$

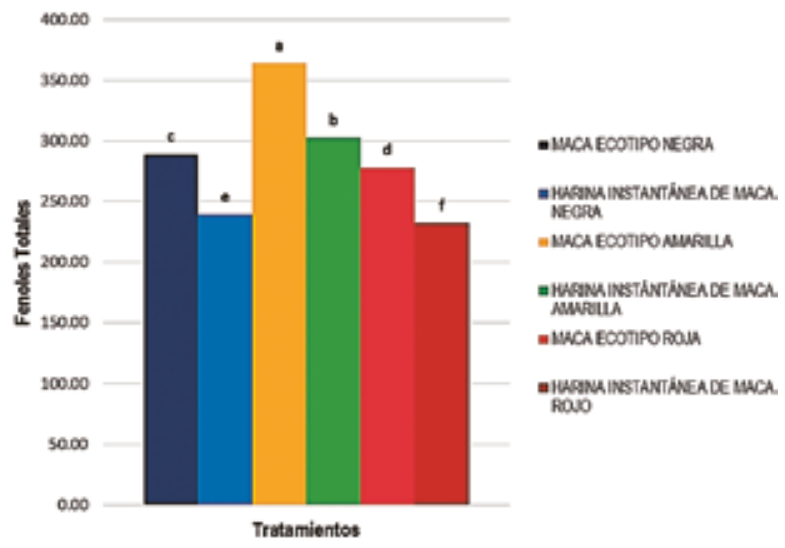

\section{Discusión}

Se determinó la capacidad antioxidante y polifenoles totales de ecotipos de maca amarilla, rojo y negro y de las harinas instantáneas de los ecotipos estudiados. El consumo de alimentos con antioxidantes nos ayuda a mantenernos sanos y prolongar el proceso biológico de envejecimiento.

El contenido de humedad, proteínas, grasas y cenizas de los ecotipos de maca (Tabla 1), es mayor que el de 
harinas instantáneas que presentan mayor contenido de carbohidratos. Los resultados son el promedio de 3 repeticiones por ecotipo y la desviación estándar de la media. El ecotipo rojo, tiene mayor contenido de humedad $(14,75 \%)$; el negro, mayor contenido de proteína $(12,78 \%)$, fibra $(4,88 \%)$ y ceniza $(5,47 \%)$; mientras que el ecotipo amarillo, presenta mayor contenido de carbohidratos $(67,99 \%)$ y grasa $(0,38 \%)$.

Estos resultados se encuentran dentro de los rangos establecidos por Obregón (1998) y Machado (2001) para la maca seca. Se encontró diferencias significativas para un nivel de significancia de $\mathrm{P}<0,05$, en los contenidos de humedad, proteína, grasa, fibra y carbohidratos en los tres ecotipos a excepción de las cenizas.

El contenido de humedad, proteínas, grasas y cenizas de los ecotipos de maca (Tabla 1), es mayor que el de harinas instantáneas que presentan mayor contenido de carbohidratos. Los resultados son el promedio de 3 repeticiones por ecotipo y la desviación estándar de la media. El ecotipo rojo, tiene mayor contenido de humedad (14,75\%); el negro, mayor contenido de proteína $(12,78 \%)$, fibra $(4,88 \%)$ y ceniza $(5,47 \%)$; mientras que el ecotipo amarillo presenta mayor contenido de carbohidratos $(67,99 \%)$ y grasa $(0,38 \%)$. Estos resultados se encuentran dentro de los rangos establecidos por Obregón (1998) y Machado (2001) para la maca seca. Se encontró diferencias significativas para un nivel de significancia de $\mathrm{P}<0,05$, en los contenidos de humedad, proteína, grasa, fibra y carbohidratos en los tres ecotipos a excepción de las cenizas.

La humedad disminuye significativamente en las harinas de los tres ecotipos (negro, amarillo y rojo) (Tabla 2), presentando valores en el rango de 4,75 a 5,31\%, las proteínas también disminuyen en pequeños porcentajes, presentado valores entre 10,29 a 11,59\%. La grasa aumenta en el ecotipo rojo y disminuye en los ecotipos amarillo y negro, mientras que la fibra y cenizas disminuye en los tres ecotipos. Estos resultados se encuentran dentro de los rangos establecidos por Rojas (2001), Torres (1984) y Vílchez, Guevara y Encina (2012). Se encontró diferencias significativas para un nivel de significancia de $\mathrm{P}<0,05$, en humedad, proteína, fibra, cenizas y carbohidratos, excepto las grasas. Los resultados son el promedio de 3 repeticiones y la desviación estándar de la media. El análisis físico químico como índice de solubilidad e índice de absorción de agua, de las harinas instantáneas de tres ecotipos de maca, presenta valores que se encuentran dentro de los rangos correspondientes a harina instantánea.

Los resultados del análisis físico químico de las harinas instantáneas de tres ecotipos de maca (Tabla 3), tales como el índice de solubilidad en harinas de maca amarilla, roja y negro fueron 14,4, 14,2 y 13,9\%, respectivamente y el índice de absorción de agua fue de 4,3,
4,2 y 4,2 para las harinas de maca amarilla, rojo y negro, respectivamente. Estos resultados de índice de absorción son considerados buenos, al estar en el rango de 1,76 a 5,47 para precocidos de maíz, el índice de solubilidad también se considera buenos, según Rodríguez et. al., (2006), al aumentar el índice de absorción con el grado de modificación del almidón. Estos resultados muestran que los gránulos de almidón de la harina extruida de maca de los tres ecotipos han sido modificados y, por lo tanto, se considera a la harina extruida de maca como una harina instantánea. La acidez titulable de las harinas de ecotipos amarillo, rojo y negro fueron: 0,38\%, $0,36 \%$ y $0,40 \%$, y los valores de $\mathrm{pH}$ fueron: 5, 56, 5,69 y 5,43 , respectivamente, estando cercano a los valores recomendados para harinas sucedáneos de la harina de trigo (Rodríguez et al., 2006). Se trabajó con harinas instantáneas de tres ecotipos de maca de grado de finura medio y fino.

En las tablas 4, 5, y 6, se muestran los resultados del análisis granulométrico de las harinas extruidas de maca amarilla, roja y negra, que presentan un módulo de finura de 3,0771, 3,0215 y 3,0078, respectivamente, encontrándose clasificado según INDECOPI dentro del grado medio, lo cual nos indica que las harinas están conformadas en su mayoría por partículas medias y en menor proporción por partículas finas (INDECOPI, 1998). La inhibición de radicales libres depende entre otros factores de la granulometría de las muestras (Zavaleta, 2005).

La capacidad antioxidante y el contenido de polifenoles totales de los ecotipos de maca es mayor que el de harinas instantáneas. Los resultados (Tabla 7), muestran que existe influencia de los tres ecotipos en la capacidad antioxidante de la harina instantánea de maca, debido a que en todos los casos (ecotipos), se observa que el \% de inhibición de radicales libres disminuye en las harinas instantáneas de maca obtenidas por extrusión. El ecotipo de maca negra y la harina instantánea de esta reportan 47,83 y 36,25 \% de inhibición, respectivamente, la maca amarilla y la harina tienen 15,46 y $14,01 \%$ de inhibición, la maca roja y la harina presen$\tan 28,95$ y 27,07 \% de inhibición.

Estos resultados comparados con los obtenidos por otros autores (Castañeda et al., 2008; Sucari et al., 2013), son menores en vista que ellos presentan un porcentaje de captación de radicales libres de la Harina de maca Koken y harina de maca Amazon con un \% de inhibición de radicales libres de 60,32\% y 40,71\% respectivamente. Estas diferencias podrían ser caudadas por el proceso y los parámetros de procesamiento de las harinas de maca, así como el tipo de extrusor utilizado y las condiciones climáticas de la zona donde produce la maca, entre otros. El análisis de variancia y prueba de Tukey de la capacidad antioxidante de ecotipos y harinas instantáneas 
de maca (Figura 2), muestra que existe diferencia significativa entre las medias de estos a un nivel de 95 $\%$ de probabilidad y, por lo tanto, se confirma que los ecotipos de maca influyen en la capacidad antioxidante de las harinas instantáneas.

Los resultados (Tabla 8), muestran que existe influencia de los tres ecotipos en el contenido de polifenoles totales de las harinas instantáneas de maca, debido a que, en todos los casos, se observa que los mg. de ácido gálico equivalente por 100 gramos de muestra, disminuye en las harinas instantáneas de maca obtenidas por extrusión. El ecotipo de maca negra y la harina instantánea de esta reportan 289 y 239,33 mg GAE/100g, respectivamente; la maca amarilla y la harina tienen 364 y 302,33 mg GAE/100g; la maca roja y la harina presentan 278 y 232,33 mg GAE/100 g. Estos resultados comparados con los obtenidos por otros autores (Doroteo et al., 2013), son menores, quienes reportan un contenido de fenólicos totales para la maca de $930 \mathrm{mg}$. GAE/100 g. de extracto. Estas diferencias podrían ser caudadas por el proceso y los parámetros de procesamiento de las harinas de maca, así como, por el tipo de extrusor utilizado, entre otros. Sin embargo, estos resultados si concuerdan con los obtenidos por otros autores (Sucari et al., 2013), quienes para una mezcla extruida (50\% de maca, $30 \%$ de cañihua y $20 \%$ de maíz morado) reportan 273 y $257 \mathrm{mg}$ de ácido gálico equivalente/100 g, trabajando a una temperatura de $120^{\circ} \mathrm{C}$ y un flujo de alimentación de 400 y $800 \mathrm{~g} / \mathrm{min}$.

El análisis de varianza y prueba de Tukey (Figura 3) del contenido de fenoles totales de ecotipos y harinas instantáneas de maca, muestra que existe diferencia significativa entre las medias a un nivel de $95 \%$ de probabilidad y, por tanto, se confirma que los ecotipos de maca influyen en el contenido de fenoles totales de las harinas instantáneas de maca, resultados que si concuerdan con los obtenidos por otros autores (Sucari et al., 2013), y son menores a los resultados obtenidos por otros autores (Doroteo et al., 2013).

La capacidad antioxidante de cada ecotipo de maca es diferente, estas diferencias podrían ser causadas por el color de cada una de ellas, por lo que su consumo y utilización debe ser diferenciada según sectores o nichos de mercado logrando promover la diversificación del cultivo de maca en los andes peruanos.

\section{Conclusiones}

- Se ha encontrado que existe influencia de los tres ecotipos de maca en la capacidad antioxidante de las harinas instantáneas obtenidas por extrusión, esto se observa en la disminución del porcentaje de inhibición de radicales libres. El contenido de polifenoles totales también es menor en las harinas procesadas.
- Los ecotipos influyen en la composición química proximal de las harinas instantáneas de maca, disminuyendo el contenido de humedad, proteínas, grasa, fibra y cenizas, aumentando el contenido de carbohidratos.

- El análisis de varianza y prueba de Tukey de la capacidad antioxidante y contenido de fenoles totales de ecotipos y harinas instantáneas de maca, permiten concluir que existe diferencia significativa entre las medias de los tratamientos a un nivel de 95\% de probabilidad y, por ende, se confirma que los ecotipos de maca influyen en la capacidad antioxidante y contenido de fenoles totales de las harinas instantáneas de maca.

- Debería elaborarse las harinas instantáneas de ecotipos de maca para mercados específicos indicando las bondades del producto.

- Utilizar otros métodos de determinación de antioxidantes para validar los resultados obtenidos en el presente estudio.

\section{Referencias bibliográficas}

Association of Official Analytical Chemists. [AOAC] (1995). Official Méthods of Analysis. 15 Ed. Washington D.C. Vol. 2. 850 pp.

Brand-Williams, W., Cuvelier, M., Berset, C. (1995). Use of a free radical method to evaluate antioxidant activity. Trends in Food Sci. and Tech., 28(3), 25-30

Campos, D., Chirinos, R., Barreto, O., Noratto, G., \& Pedreschi, R. (2013). Optimized methodology for the simultaneous extraction of glucosinolates, phenolic compounds and antioxidant capacity from maca (Lepidium meyenii). Industrial Crops and Products, 49, 747-754. https://doi.org/10.1016/J. INDCROP.2013.06.021

Castañeda, C., Ramos, E., Ibáñez, L. Evaluación de la capacidad antioxidante de siete plantas medicinales peruanas. Horizonte Médico [en línea] 2008, 8 (Junio - Sin mes): [Fecha de consulta: 9 de diciembre de 2018] Disponible en: http: / / www.redalyc.org/ articulo.oa?id=371637117004ISSN1727-558X

Doroteo, Víctor Hugo, Díaz, Camilo, Terry, Cecilia, \& Rojas, Rosario. (2013). Compuestos fenólicos y actividad antioxidante in vitro de 6 plantas peruanas. Revista de la Sociedad Química del Perú, 79(1), 13-20. Recuperado en 19 de diciembre de 2018, de http: / / www.scielo.org.pe/scielo.php?script $=_{\text {s- }}$ ci_arttext\&pid=S1810-634X2013000100003\&l$\mathrm{ng}=\mathrm{es} \& \mathrm{t} \operatorname{lng}=\mathrm{es}$.

Durand V., Lavado B., Napán M., Palomino M. (2004). 
Estudio de pre factibilidad para la instalación de una planta procesadora de harina y tabletas de maca para la exportación a Estados Unidos y Japón. Ciclo optativo de profesionalización en Gestión Agrícola Empresarial. Universidad Nacional Agraria La Molina. Lima - Perú.

Gonzáles, C., Rubio, J., Gasco, M., Nieto, J., Yucra, S., \& Gonzáles, G. f. (2006). Effect of short-term and long-term treatments with three ectypes of lepidium meyenii (MACA) on spermatogenesis in rats. Journal of Ethnopharmacology, 103(3), 448-454. https: / / doi.org/https://doi.org/10.1016/j.jep. 2005.08.035

INDECOPI (1998). Norma Técnica Peruana: cereales y harinas-Lima, Perú.

Javier Sucari, P. J., Lima Hurtado, M. (2013). Efecto del proceso de cocción - Extrusión en la estabilidad de los compuestos bioactivos y capacidad antiradicalaria en un alimento a base de cañihua (Chenopodium pallidicaule aellen), maca (Lepidium meyenni walp) y maíz morado (Zea mayz L.). Tesis Universidad Nacional de San Antonio Abad del Cusco.

Machado M.R. (2001). Caracterización física química de 4 ecotipos de maca (Lepidium meyenii walp). Procesos de liofilización, atomización y pre gelatinización en el ecotipo seleccionado". Tesis UNAS, Tingo María-Perú.

Obregón, V.L. (1998). Maca - planta medicinal y nutritiva del Perú. Primera Edición. Instituto de Fitoterapia Americano - Lima (Perú).

Pascual CH. G y Ramos V.C (2000). Manual de Prácticas de tecnología de cereales y leguminosas. Folleto Universidad Nacional Agraria La Molina - Facultad de Ingeniería en Industrias Alimentarias. Lima, Perú.

Rodríguez E., Fernández A., Alonso L.; y Ospina B. (2006). Reología de suspensiones preparadas con harina precocida de yuca. Ingeniería \& Desarrollo número 19 enero-junio, 2006 ISSN: 0122-3461. En línea: http://rcientificas.uninorte.edu.co/index.php/ingenieria/article/viewFile/2479/1624

Rojas R.S. (2001). Estudio Técnico para la obtención de harina de maca (Lepidium meyenii walp) Extruida. Tesis Universidad Nacional del Centro del Perú, Facultad de Ingeniería en Industrias Alimentarias, Huancayo - Perú.

Singleton, V. y Rossi J. Colorimetry of total phenolics ans phosphomolybdic-phosphotungstic acid reagents. Am.J. Enol.Vitic. 1965; 16, 144-158 p.

Sun, Y., Dai, C., Shi, S., Zheng, Y., Wei, W., \& Cai, D. (2008). Composition analysis and antioxidant ac- tivity of essential oils, lipids and polysaccharides in different phenotypes of Lepidium meyenii. Journal of Chromatography B, 1099, 25-33. https://doi.org/ https://doi.org/10.1016/j.jchromb. 2018.09.10

Torres, V.R. (1984). Estudio Nutricional de la Maca (Lepidium meyenii walp) y su aplicación en la Elaboración de una bebida base. Tesis UNA. La Molina Lima - Perú.

Troya-Santos, J., Ale-Borja, N., Suárez-CunzA, S. (2017). In vitro antioxidant capacity of traditional prepared black maca (Lepidium meyenii). Revista de la Sociedad Química del Perú, 83(1), 40-51. Recuperado en 12 de marzo de 2018, de http:// www.scielo.org.pe/scielo.php?script $=$ sci_arttext\&pid $=$ S $1810-634$ X2017000100005\&l$\mathrm{ng}=\mathrm{es} \& \mathrm{t} \operatorname{lng}=\mathrm{en}$.

Vílchez L.M., Guevara A., Encina C. R. Influencia del tamaño de partícula, humedad y temperatura en el grado de gelatinización durante el proceso de extrusión de maca (Lepidium meyenii Walp) Rev. Soc. Quím. Perú v.78 n.2 Lima abr./jun. 2012.

Vivas, B.M (1979). Estudio técnico para la obtención de una mezcla vegetal básica precocida para consumo humano. Tesis para optar el título de Ingeniero en Industrias Alimentarias, Universidad Agraria La Molina. LIMA, Perú.

Yábar, E., Pedreschi, R., Chirinos, R., \& Campos, D. (2011). Glucosinolate content and myrosinase activity evolution in three maca (Lepidium meyenii walp) ecotypes during preharvest, harvest and postharvest drying. Food Chemistry, 127 (4), 1576-1583. https://doi.org/10.1016/j.foodchem.2011.02.021.

Zavaleta J. 2005. Capacidad antioxidante y determinación de ácidos fenólicos y flavonoides en 8 alimentos nativos del Perú. [Tesis de postgrado]. Lima: Escuela de Post Grado-Especialidad de Bioquímica y Nutrición, Facultad de Medicina Humana, Universidad Privada de San Martín de Porres. 